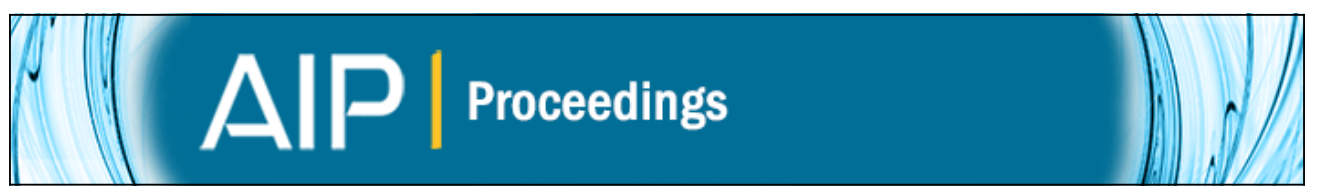

\title{
GRB Cosmology: Probing the Early Universe
}

Volker Bromm and Abraham Loeb

Citation: AIP Conference Proceedings 937, 532 (2007); doi: 10.1063/1.3682957

View online: http://dx.doi.org/10.1063/1.3682957

View Table of Contents: http://scitation.aip.org/content/aip/proceeding/aipcp/937?ver=pdfcov

Published by the AIP Publishing

Articles you may be interested in

GRB Probes of the Early Universe with EXIST

AIP Conf. Proc. 1279, 212 (2010); 10.1063/1.3509267

Cosmology of the Very Early Universe

AIP Conf. Proc. 1268, 3 (2010); 10.1063/1.3483879

Early Universe cosmology with mirror dark matter

AIP Conf. Proc. 1241, 351 (2010); 10.1063/1.3462655

Cyclical behavior in early universe cosmologies

J. Math. Phys. 41, 6277 (2000); 10.1063/1.1286878

Qualitative analysis of early universe cosmologies

J. Math. Phys. 40, 5092 (1999); 10.1063/1.533017 


\title{
GRB Cosmology: Probing the Early Universe
}

\author{
Volker Bromm* and Abraham Loeb ${ }^{\dagger}$ \\ *Astronomy Department, University of Texas, Austin, TX 78712 \\ ${ }^{\dagger}$ Astronomy Department, Harvard University, Cambridge, MA 02138
}

\begin{abstract}
.
Current observations are about to open up a direct observational window into the final frontier of cosmology: the crucial first billion years in cosmic history when the first stars and galaxies formed. Even before the launch of the James Webb Space Telescope, it would be possible to utilize GammaRay Bursts (GRBs) as unique probes of cosmic star formation and the state of the intergalactic medium up to redshifts of the first stars. The ongoing $S$ wift mission might be the first observatory to detect individual Population III stars, provided that massive metal-free stars were able to trigger GRBs. Swift will empirically constrain the redshift at which Population III star formation was terminated, thus providing crucial input to models of cosmic reionization and metal enrichment.
\end{abstract}

Keywords: cosmology: theory - early universe - gamma rays: bursts

PACS: $95.30 . \mathrm{Lz} ;$ 97.10.Bt; 97.20.Wt; $98.70 . \mathrm{Rz}$

\section{INTRODUCTION}

Gamma-Ray Bursts (GRBs) are believed to originate in compact remnants (neutron stars or black holes) of massive stars. Their high luminosities make them detectable out to the edge of the visible universe [1,2]. GRBs offer the opportunity to detect the most distant (and hence earliest) population of massive stars, the so-called Population III (Pop III henceforth), one star at a time. In the hierarchical assembly process of halos which are dominated by cold dark matter (CDM), the first galaxies should have had lower masses (and lower stellar luminosities) than their low-redshift counterparts. Consequently, the characteristic luminosity of galaxies or quasars is expected to decline with increasing redshift. GRB afterglows, which already produce a peak flux comparable to that of quasars or starburst galaxies at $z \sim 1-2$, are therefore expected to outshine any competing source at the highest redshifts, when the first dwarf galaxies have formed in the universe.

The polarization data from the Wilkinson Microwave Anisotropy Probe (WMAP) indicates an optical depth to electron scattering of $\sim 9 \pm 3 \%$ after cosmological recombination [3]. This implies that the first stars must have formed at a redshift $z \sim 20$, and reionized a substantial fraction of the intergalactic hydrogen around that time $[4,5,6,7,8]$. Early reionization can be achieved with plausible star formation parameters in the standard $\Lambda \mathrm{CDM}$ cosmology; in fact, the required optical depth can be achieved in a variety of very different ionization histories since $W M A P$ places only an integral constraint on these histories [9]. One would like to probe the full history of reionization in order to disentangle the properties and formation history of the stars that are responsible for it. GRB afterglows offer the opportunity to detect stars as well as to probe the ionization state [10] and metal enrichment level [11] of the intervening intergalactic medium (IGM). 


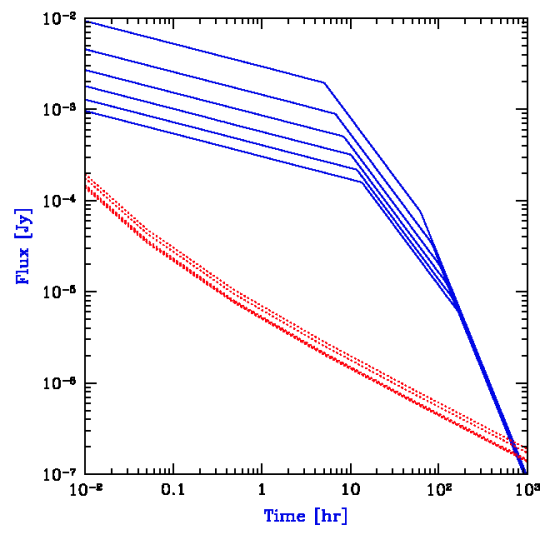

FIGURE 1. Detectability of high-redshift GRB afterglows as a function of time since the GRB explosion as measured by the observer (from [10]). The GRB afterglow flux is shown (solid curves) at the redshifted Ly $\alpha$ wavelength. Also shown (dotted curves) is the detection threshold for JWST assuming a spectral resolution $R=5000$ with the near infrared spectrometer, a signal to noise ratio of 5 per spectral resolution element, and an exposure time equal to $20 \%$ of the time since the GRB explosion. In each set of curves, a sequence of redshifts is used, $z=5,7,9,11,13$, and 15, respectively, from top to bottom.

GRBs, the electromagnetically-brightest explosions in the universe, should be detectable out to redshifts $z>10[1,2]$. High-redshift GRBs can be identified through infrared photometry, based on the Ly $\alpha$ break induced by absorption of their spectrum at wavelengths below $1.216 \mu \mathrm{m}[(1+z) / 10]$. Follow-up spectroscopy of high-redshift candidates can then be performed on a 10-meter-class telescope. Recently, the ongoing Swift mission [12] has detected a GRB originating at $z \simeq 6.3$ (e.g., [13]), thus demonstrating the viability of GRBs as probes of the early universe.

There are four main advantages of GRBs relative to traditional cosmic sources such as quasars:

(i) The GRB afterglow flux at a given observed time lag after the $\gamma$-ray trigger is not expected to fade significantly with increasing redshift, since higher redshifts translate to earlier times in the source frame, during which the afterglow is intrinsically brighter [1]. For standard afterglow lightcurves and spectra, the increase in the luminosity distance with redshift is nearly compensated by this cosmological time-stretching effect. This is illustrated in Figure 1.

(ii) As already mentioned, in the standard $\Lambda \mathrm{CDM}$ cosmology, galaxies form hierarchically, starting from small masses and increasing their average mass with cosmic time. Hence, the characteristic mass of quasar black holes and the total stellar mass of a galaxy were smaller at higher redshifts, making these sources intrinsically fainter [14]. However, GRBs are believed to originate from a stellar mass progenitor and so the intrinsic luminosity of their engine should not depend on the mass of their host galaxy. GRB afterglows are therefore expected to outshine their host galaxies by a factor that gets larger with increasing redshift.

(iii) Since the progenitors of GRBs are believed to be stellar, they likely originate in the 
most common star-forming galaxies at a given redshift rather than in the most massive host galaxies, as is the case for bright quasars [10]. Low-mass host galaxies induce only a weak ionization effect on the surrounding IGM and do not greatly perturb the Hubble flow around them. Hence, the Ly $\alpha$ damping wing should be closer to the idealized unperturbed IGM case and its detailed spectral shape should be easier to interpret. Note also that unlike the case of a quasar, a GRB afterglow can itself ionize at most $\sim 4 \times 10^{4} E_{51} M_{\odot}$ of hydrogen if its UV energy is $E_{51}$ in units of $10^{51} \mathrm{ergs}$ (based on the available number of ionizing photons), and so it should have a negligible cosmic effect on the surrounding IGM.

(iv) GRB afterglows have smooth (broken power-law) continuum spectra unlike quasars which show strong spectral features (such as broad emission lines or the so-called "blue bump") that complicate the extraction of IGM absorption features. In particular, the continuum extrapolation into the $\operatorname{Ly} \alpha$ damping wing (the Gunn-Peterson absorption trough) during the epoch of reionization is much more straightforward for the smooth UV spectra of GRB afterglows than for quasars with an underlying broad Ly $\alpha$ emission line [10].

Although the nature of the central engine that powers the relativistic jets of GRBs is still unknown, recent evidence indicates that long-duration GRBs trace the formation of massive stars (e.g., $[15,16,17,18,19,20]$ ) and in particular that long-duration GRBs are associated with Type Ib/c supernovae [21]. Since the first stars in the universe are predicted to be predominantly massive $[22,23,24]$, their death might give rise to large numbers of GRBs at high redshifts. In contrast to quasars of comparable brightness, GRB afterglows are short-lived and release $\sim 10$ orders of magnitude less energy into the surrounding IGM. Beyond the scale of their host galaxy, they have a negligible effect on their cosmological environment. Note, however, that feedback from a single GRB or supernova on the gas confined within early dwarf galaxies could be dramatic, since the binding energy of most galaxies at $z>10$ is lower than $10^{51}$ ergs [25]. Consequently, they are ideal probes of the IGM during the reionization epoch. Their rest-frame UV spectra can be used to probe the ionization state of the IGM through the spectral shape of the Gunn-Peterson (Ly $\alpha$ ) absorption trough, or its metal enrichment history through the intersection of enriched bubbles of supernova (SN) ejecta from early galaxies [11]. Afterglows that are unusually bright $(>10 \mathrm{mJy})$ at radio frequencies should also show a detectable forest of $21 \mathrm{~cm}$ absorption lines due to enhanced HI column densities in sheets, filaments, and collapsed minihalos within the IGM [26].

Another advantage of GRB afterglows is that once they fade away, one may search for their host galaxies. Hence, GRBs may serve as signposts of the earliest dwarf galaxies that are otherwise too faint or rare on their own for a dedicated search to find them. Detection of metal absorption lines from the host galaxy in the afterglow spectrum, offers an unusual opportunity to study the physical conditions (temperature, metallicity, ionization state, and kinematics) in the interstellar medium of these high-redshift galaxies. A small fraction $(\sim 10)$ of the GRB afterglows are expected to originate at redshifts $z>5[27,28]$. This subset of afterglows can be selected photometrically using a small telescope, based on the Ly $\alpha$ break at a wavelength of $1.216 \mu \mathrm{m}[(1+z) / 10]$, caused by intergalactic HI absorption. The challenge in the upcoming years will be to follow-up on these candidates spectroscopically, using a large (10-meter class) telescope. GRB afterglows are likely to revolutionize observational cosmology and replace traditional 
sources like quasars, as probes of the IGM at $z>5$. The near future promises to be exciting for GRB astronomy as well as for studies of the high-redshift universe.

\section{KEY QUESTIONS IN GRB COSMOLOGY}

Using GRBs to probe the high redshift universe has great promise. In the following, we discuss some key open questions.

\section{Cosmic Star Formation at High Redshifts}

It is of great importance to constrain the Pop III star formation mode, and in particular to determine down to which redshift it continues to be prominent. The extent of the Pop III star formation will affect models of the initial stages of reionization (e.g., $[7,5,29,8,30])$ and metal enrichment (e.g., [31, 11, 32, 33, 34]), and will determine whether planned surveys will be able to effectively probe Pop III stars (e.g., [35]). The constraints on Pop III star formation will also determine whether the first stars could have contributed a significant fraction to the cosmic near-IR background (e.g., $[36,37,38,39,40])$.

To constrain high-redshift star formation, one has to carry out a two-step approach: (1) What is the signature of GRBs that originate in metal-free, Pop III progenitors? Simply knowing that a given GRB came from a high redshift is not sufficient to reach a definite conclusion as to the nature of the progenitor. E.g., the currently highestredshift GRB at $z \simeq 6.3$ clearly did not originate from a Pop III progenitor, given the inferred level of metal enrichment in the host system of a few percent solar (e.g., [41]). Pregalactic metal enrichment was likely quite inhomogeneous, and we expect normal Pop I and II stars to exist in galaxies that were already metal-enriched at these high redshifts [28]. Pop III and Pop I/II star formation is thus predicted to have occurred concurrently at $z>5$. How is the predicted high mass-scale for Pop III stars reflected in the observational signature of the resulting GRBs? Our preliminary results indicate that circumburst densities are systematically higher in Pop III environments. GRB afterglows will then be much brighter than for conventional GRBs. In addition, due to the systematically increased progenitor masses, the Pop III distribution may be biased toward long-duration events.

(2) The modelling of Pop III cosmic star formation histories has a number of free parameters, such as the star formation efficiency and the strength of the chemical feedback. The latter refers to the timescale for, and spatial extent of, the distribution of the first heavy elements that were produced inside of Pop III stars, and subsequently dispersed into the IGM by supernova blast waves. Comparing with theoretical GRB redshift distributions one can use the GRB redshift distribution observed by Swift to calibrate the free model parameters. In particular, one can use this strategy to measure the redshift where Pop III star formation terminates.

Figure 2 illustrates this approach (based on [28]) and leads to the robust expectation that $\sim 10 \%$ of all Swift bursts should originate at $z>5$. This prediction is based on the contribution from Population I/II stars which are known to exist even at these 


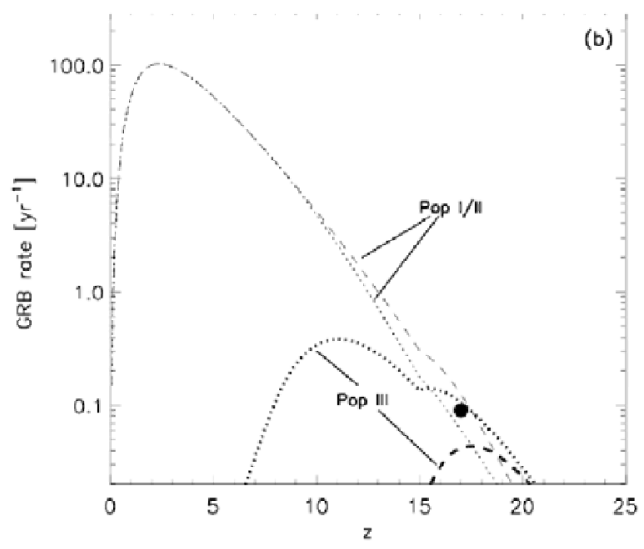

FIGURE 2. Predicted GRB rate to be observed by $S$ wift (from [28]). Shown is the observed number of bursts per year, $d N_{\mathrm{GRB}}^{\mathrm{obs}} / d \ln (1+z)$, as a function of redshift. All rates are calculated with a constant GRB efficiency, $\eta_{\mathrm{GRB}} \simeq 2 \times 10^{-9}$ bursts $M_{\odot}^{-1}$, using a theoretically calculated cosmic star formation rate. Dotted lines: Contribution to the observed GRB rate from Pop I/II and Pop III for the case of weak chemical feedback. Dashed lines: Contribution to the GRB rate from Pop I/II and Pop III for the case of strong chemical feedback. Filled circle: GRB rate from Pop III stars if these were responsible for reionizing the universe at $z \sim 17$.

high redshifts. Additional GRBs could be triggered by Pop III stars, with a highly uncertain efficiency. Assuming that long-duration GRBs are produced by the collapsar mechanism, a Pop III star with a close binary companion provides a plausible GRB progenitor, leading to a rapidly spinning black hole. Recently, however, single star progenitors have been suggested [42], and the binary pathway to GRBs from Pop III stars has been critically analyzed [43]. Based on the binary model, one can estimate [28] the Pop III GRB efficiency, reflecting the probability of forming sufficiently close and massive binary systems, to lie between zero (if tight Pop III binaries do not exist) and $\sim 10$ times the empirically inferred value for Population I/II (due to the increased fraction of black hole forming progenitors among the massive Population III stars).

A key ingredient in determining the underlying star formation history from the observed GRB redshift distribution is the GRB luminosity function, which is only poorly constrained at present. The improved statistics provided by Swift will enable the construction of an empirical luminosity function. With an improved luminosity function, we will be able to re-calibrate the theoretical prediction in Figure 2 more reliably.

\section{Physical Properties of GRB Hosts}

In order to predict the observational signature of high-redshift GRBs, it is important to know the properties of the GRB host systems. Within variants of the popular CDM model for structure formation, where small objects form first and subsequently merge to build up more massive ones, the first stars are predicted to form at $z \sim 20-30$ in 

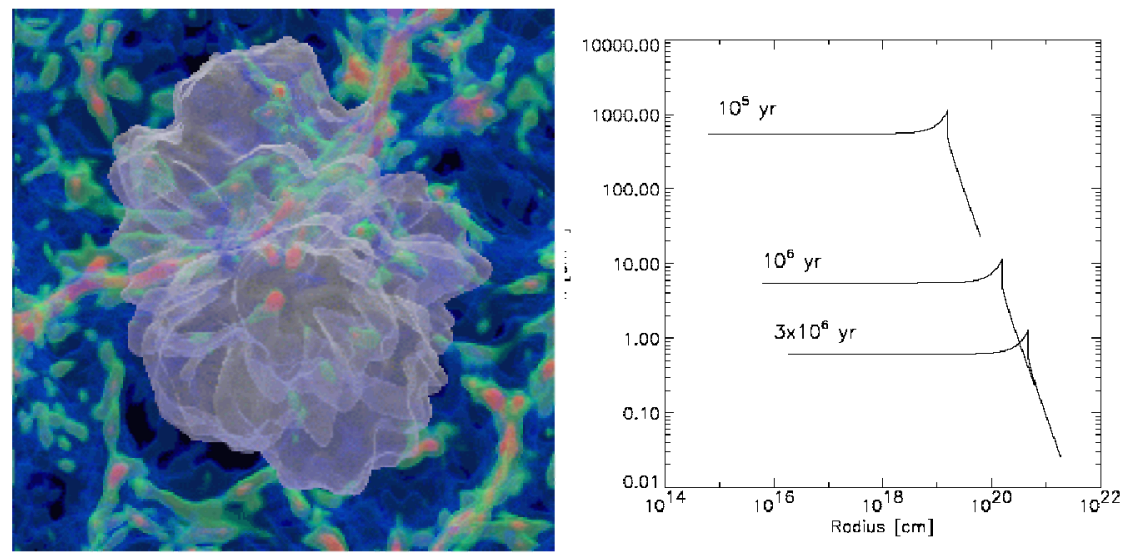

FIGURE 3. Radiative feedback in the high-redshift universe. Left panel: We show a cosmological box at $z \simeq 20$ with a (proper) linear size of $\sim 13.6 \mathrm{kpc}$ (adopted from [30]). In the most massive minihalo a single Pop III star with $M_{+}=200 M_{\odot}$ has formed, producing ionizing radiation for $\sim 2 \times 10^{6} \mathrm{yr}$. We superimpose the resulting H II region (translucent white surface) onto the neutral gas density field. The ionized region grows to a size with (proper) radius $\sim 5 \mathrm{kpc}$. Right panel: Effect of photoheating from a Population III star on the density profile in a high-redshift minihalo. The curves, labeled by the time after the onset of the central point source, are calculated according to a self-similar model for the expansion of an H II region. Numerical simulations closely conform to this analytical behavior. Notice that the central density is significantly reduced by the end of the life of a massive star, and that a central core has developed where the density is constant.

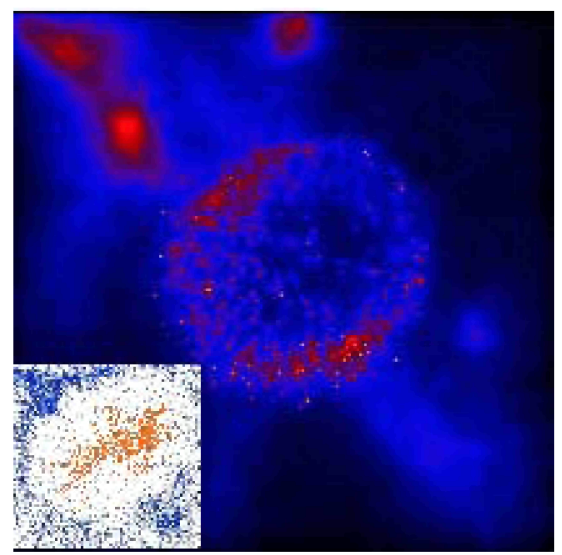

FIGURE 4. Supernova explosion in the high-redshift universe (from [48]). The snapshot is taken $\sim 10^{6} \mathrm{yr}$ after the explosion with total energy $E_{\mathrm{SN}} \simeq 10^{53} \mathrm{ergs}$. We show the projected gas density within a box of linear size $1 \mathrm{kpc}$. The SN bubble has expanded to a radius of $\sim 200 \mathrm{pc}$, having evacuated most of the gas in the minihalo. Inset: Distribution of metals. The stellar ejecta (gray dots) trace the metals and are embedded in pristine metal-poor gas (black dots). 
minihalos of total mass (dark matter plus gas) $\sim 10^{6} M_{\odot}[44,25,8]$. These objects are the sites for the formation of the first stars, and thus are the potential hosts of the highest-redshift GRBs. What is the environment in which the earliest GRBs and their afterglows did occur? This problem breaks down into two related questions: (i) what type of stars (in terms of mass, metallicity, and clustering properties) will form in each minihalo?, and (ii) how will the ionizing radiation from each star modify the density structure of the surrounding gas? These two questions are fundamentally intertwined. The ionizing photon production strongly depends on the stellar mass, which in turn is determined by how the accretion flow onto the growing protostar proceeds under the influence of this radiation field. In other words, the assembly of the Population III stars and the development of an HII region around them proceed simultaneously, and affect each other. As a first step (see Figure 3), we describe the photo-evaporation as a selfsimilar champagne flow [45], with parameters appropriate for the Population III case.

Notice that the central density is significantly reduced by the end of the life of a massive star, and that a central core has developed where the density is nearly constant. Such a flat density profile is markedly different from that created by stellar winds $\left(\rho \propto r^{-2}\right)$. Winds, and consequently mass-loss, may not be important for massive Population III stars $[46,47]$, and such a flat density profile may be characteristic of GRBs that originate from metal-free Population III progenitors.

We have carried out idealized simulations of the protostellar accretion problem, allowing us to estimate the final mass of a Population III star [49]. Using the smoothed particle hydrodynamics (SPH) method, we have included the chemistry and cooling physics relevant for the evolution of metal-free gas (see [23] for details). Improving on earlier work $[50,23]$ by initializing our simulations according to the $\Lambda \mathrm{CDM}$ model, we have focused on an isolated overdense region that corresponds to a $3 \sigma$-peak [49]: a halo containing a total mass of $10^{6} M_{\odot}$, and collapsing at a redshift $z_{\text {vir }} \simeq 20$.

We have found that one high-density clump has formed at the center of the minihalo, possessing a gas mass of a few hundred solar masses. Soon after its formation, the clump becomes gravitationally unstable and undergoes runaway collapse. Once the gas has exceeded a threshold density of $10^{7} \mathrm{~cm}^{-3}$, a sink particle is inserted into the simulation. This choice for the density threshold ensures that the local Jeans mass is resolved throughout the simulation. The clump (i.e., sink particle) has an initial mass of $M_{\mathrm{C} 1} \simeq 200 M_{\odot}$, and grows subsequently by ongoing accretion of surrounding gas. High-density clumps with such masses result from the chemistry and cooling rate of molecular hydrogen, $\mathrm{H}_{2}$, which imprint characteristic values of temperature, $T \sim 200 \mathrm{~K}$, and density, $n \sim 10^{4} \mathrm{~cm}^{-3}$, into the metal-free gas [23]. Evaluating the Jeans mass for these characteristic values results in $M_{J} \sim$ a few $\times 10^{2} M_{\odot}$, which is close to the initial clump masses found in the simulations.

The high-density clumps are clearly not stars yet. To probe the subsequent fate of a clump, we have re-simulated the evolution of the central clump with sufficient resolution to follow the collapse to higher densities (see $[51,52]$ for a description of the refinement technique). Our refined simulation enables us to study the three-dimensional accretion flow around the protostar (see also $[53,54,55,56]$ ). We now allow the gas to reach densities of $10^{12} \mathrm{~cm}^{-3}$ before being incorporated into a central sink particle. At these high densities, three-body reactions [57] have converted the gas into a fully molecular form. We follow the growth of the molecular core over the first $\sim 10^{4} \mathrm{yr}$ after its 
formation, making the idealized assumption that the protostellar radiation does not affect the accretion flow. The accretion rate is initially very high, $\dot{M}_{\text {acc }} \sim 0.1 M_{\odot} \mathrm{yr}^{-1}$, and subsequently declines roughly as a power law of time. The mass of the molecular core, taken as a crude estimate for the protostellar mass, grows approximately as: $M_{*} \sim \int \dot{M}_{\text {acc }} \mathrm{d} t \simeq 0.8 M_{\odot}(t / 1 \mathrm{yr})^{0.45}$. A robust upper limit for the final mass of the star is then: $M_{*}\left(t=3 \times 10^{6} \mathrm{yr}\right) \sim 500 M_{\odot}$. In deriving this upper bound, we have conservatively assumed that accretion cannot go on for longer than the total lifetime of a massive star.

Our numerical results can be understood within the general theoretical framework of how stars form [58]. Star formation typically proceeds from the 'inside-out', through the accretion of gas onto a central hydrostatic core. Whereas the initial mass of the hydrostatic core is very similar for primordial and present-day star formation [59], the accretion process - ultimately responsible for setting the final stellar mass - is expected to be rather different. On dimensional grounds, the accretion rate is simply related to the sound speed cubed over Newton's constant (or equivalently given by the ratio of the Jeans mass and the free-fall time): $\dot{M}_{\text {acc }} \sim c_{s}^{3} / G \propto T^{3 / 2}$. A simple comparison of the temperatures in present-day star forming regions $(T \sim 10 \mathrm{~K})$ with those in primordial ones $(T \sim 200-300 \mathrm{~K})$ already indicates a difference in the accretion rate of more than two orders of magnitude.

Can a Population III star ever reach this asymptotic mass limit? The answer to this question is not yet known with any certainty, and it depends on whether the accretion from a dust-free envelope is eventually terminated by feedback from the star (e.g., $[53,54,55,60,56])$. The standard mechanism by which accretion may be terminated in metal-rich gas, namely radiation pressure on dust grains [61], is obviously not effective for gas with a primordial composition. Recently, it has been speculated that accretion could instead be turned off through the formation of an HII region [60], or through the radiation pressure exerted by trapped Ly $\alpha$ photons [56]. The termination of the accretion process defines the current unsolved frontier in studies of Population III star formation.

The first galaxies may be surrounded by a shell of highly enriched material that was carried out in a SN-driven wind (see Fig. 4). A GRB in that galaxy may show strong absorption lines at a velocity separation associated with the wind velocity. Simulating these winds and calculating the absorption profile in the featureless spectrum of a GRB afterglow, will allow us to use the observed spectra of high-z GRBs to directly probe the degree of metal enrichment in the vicinity of the first star forming regions (see [11] for a semi-analytic treatment).

As the early afterglow radiation propagates through the interstellar environment of the GRB, it will likely modify the gas properties close to the source; these changes could in turn be noticed as time-dependent spectral features in the spectrum of the afterglow and used to derive the properties of the gas cloud (density, metal abundance, and size). The UV afterglow radiation can induce detectable changes to the interstellar absorption features of the host galaxy [62]; dust destruction could have occurred due to the GRB $X$-rays [63, 64], and molecules could have been destroyed near the GRB source [65]. Quantitatively, all of the effects mentioned above strongly depend on the exact properties of the gas in the host system. 


\section{Population III Progenitors}

Most studies to date have assumed a constant efficiency of forming GRBs per unit mass of stars. This simplifying assumption may either lead to an overestimation or underestimation of the frequency of GRBs. Metal-free stars are thought to be massive $[22,23]$ and their extended envelopes may suppress the emergence of relativistic jets out of their surface (even if such jets are produced through the collapse of their core to a spinning black hole). On the other hand, low-metallicity stars are expected to have weak winds with little angular momentum loss during their evolution, and so they may preferentially yield rotating central configurations that make GRB jets after core collapse.

What kind of metal-free, Pop III progenitor stars may lead to GRBs? Long-duration GRBs appear to be associated with Type Ib/c supernovae [21], namely progenitor massive stars that have lost their hydrogen envelope. This requirement is explained theoretically in the collapsar model, in which the relativistic jets produced by core collapse to a black hole are unable to emerge relativistically out of the stellar surface if the hydrogen envelope is retained [66]. The question then arises as to whether the lack of metal line-opacity that is essential for radiation-driven winds in metal-rich stars, would make a Pop III star retain its hydrogen envelope, thus quenching any relativistic jets and GRBs.

Aside from mass transfer in a binary system, individual Pop III stars could lose their hydrogen envelope due to either: (i) violent pulsations, particularly in the mass range $100-140 M_{\odot}$, or (ii) a wind driven by helium lines. The outer stellar layers are in a state where gravity only marginally exceeds radiation pressure due to electronscattering (Thomson) opacity. Adding the small, but still non-negligible contribution from the bound-free opacity provided by singly-ionized helium, may be able to unbind the atmospheric gas. Therefore, mass-loss might occur even in the absence of dust, or any heavy elements.

\section{REFERENCES}

1. Ciardi, B., \& Loeb, A. 2000, ApJ, 540, 687

2. Lamb, D. Q., \& Reichart, D. E. 2000, ApJ, 536, 1

3. Spergel, D. N., et al. 2007, ApJ, in press (astro-ph/0603449)

4. Cen, R. 2003, ApJ, 591, L5

5. Ciardi, B., Ferrara, A., \& White, S.D.M. 2003, MNRAS, 344, L7

6. Somerville, R. S., \& Livio, M. 2003, ApJ, 593, 611

7. Wyithe, J. S. B., \& Loeb, A. 2003, ApJ, 588, L69

8. Yoshida, N., Bromm, V., \& Hernquist, L. 2004, ApJ, 605, 579

9. Haiman, Z., \& Holder, G. P. 2003, ApJ, 595, 1

10. Barkana, R., \& Loeb, A. 2004, ApJ, 601, 64

11. Furlanetto, S. R., \& Loeb, A. 2003, ApJ, 588, 18

12. Gehrels, N., et al. 2004, ApJ, 611, 1005

13. Haislip, J., et al. 2006, Nature, 440, 181

14. Wyithe, J. S. B., \& Loeb, A. 2002, ApJ, 581, 886

15. Totani, T. 1997, ApJ, 486, L71

16. Wijers, R.A.M.J., Bloom, J.S., Bagla, J.S., Totani, T. 1997, ApJ, 486, L71 1998, MNRAS, 294, L13

17. Blain, A. W., \& Natarajan, P. 2000, MNRAS, 312, L35

18. Kulkarni, S. R., et al. 2000, Proc. SPIE, 4005,9 
19. Bloom, J. S., Kulkarni, S. R., \& Djorgovski, S. G. 2002, AJ, 123, 1111

20. Natarajan, P., Albanna, B., Hjorth, J., Ramirez-Ruiz, E., Tanvir, N., \& Wijers, R.A.M.J. 2005, MNRAS, 364, L8

21. Stanek, K. Z., et al. 2003, ApJ, 591, L17

22. Abel, T., Bryan, G., \& Norman, M. L. 2002, Science, 295, 93

23. Bromm, V., Coppi, P. S., \& Larson, R. B. 2002, ApJ, 564, 23

24. Bromm, V., \& Larson, R. B. 2004, ARAA, 42, 79

25. Barkana, R., \& Loeb, A. 2001, Phys. Rep., 349, 125

26. Furlanetto, S. R., \& Loeb, A. 2002, ApJ, 579, 1

27. Bromm, V., \& Loeb, A. 2002, ApJ, 575, 111

28. Bromm, V., \& Loeb, A. 2006, ApJ, 642, 382

29. Sokasian, A., Yoshida, N., Abel, T., Hernquist, L., \& Springel, V. 2004, MNRAS, 350, 47

30. Alvarez, M. A., Bromm, V., \& Shapiro, P. R. 2006, ApJ, 639, 621

31. Mackey, J., Bromm, V., \& Hernquist, L. 2003, ApJ, 586, 1

32. Furlanetto, S. R., \& Loeb, A. 2005, ApJ, 634, 1

33. Schaye, J., Aguirre, A., Kim, T.-S., Theuns, T., Rauch, M., \& Sargent, W. L. W. 2003, ApJ, 596, 768

34. Simcoe, R. A., Sargent, W. L. W., \& Rauch, M. 2004, ApJ, 606, 92

35. Scannapieco, E., Madau, P., Woosley, S., Heger, A., \& Ferrara, A. 2005, ApJ, 633, 1031

36. Santos, M. R., Bromm, V., \& Kamionkowski, M. 2002, MNRAS, 336, 1082

37. Salvaterra, R., \& Ferrara, A. 2003, MNRAS, 339, 973

38. Kashlinsky, A., Arendt, R. G., Mather, J., \& Moseley, S. H. 2005, Nature, 438, 45

39. Madau, P., \& Silk, J. 2005, MNRAS, 359, L37

40. Dwek, E., Arendt, R. G., \& Krennrich, F. 2005, ApJ, 635, 784

41. Campana, S., et al. 2007, ApJ, 654, L17

42. Woosley, S. E., \& Heger, A. 2006, ApJ, 637, 914

43. Belczynski, K., Bulik, T., Heger, A., \& Fryer, C. L. 2007, ApJ, in press (astro-ph/0610014)

44. Tegmark, M., Silk, J., Rees, M. J., Blanchard, A., Abel, T., \& Palla, F. 1997, ApJ, 474, 1

45. Shu, F. H., Lizano, S., Galli, D., Cantó, J., \& Laughlin, G. 2002, ApJ, 580, 969

46. Baraffe, I., Heger, A., \& Woosley, S. E. 2001, ApJ, 550, 890

47. Kudritzki, R. P. 2002, ApJ, 577, 389

48. Bromm, V., Yoshida, N., \& Hernquist, L. 2003, ApJ, 596, L135

49. Bromm, V., \& Loeb, A. 2004, NewA, 9, 353

50. Bromm, V., Coppi, P. S., \& Larson, R. B. 1999, ApJ, 527, L5

51. Kitsionas, S., \& Whitworth, A. P. 2002, MNRAS, 330, 129

52. Bromm, V., \& Loeb, A. 2003, ApJ, 596, 34

53. Omukai, K., \& Palla, F. 2001, ApJ, 561, L55

54. Omukai, K., \& Palla, F. 2003, ApJ, 589, 677

55. Ripamonti, E., Haardt, F., Ferrara, A., \& Colpi, M. 2002, MNRAS, 334, 401

56. Tan, J. C., \& McKee, C. F. 2004, ApJ, 603, 383

57. Palla, F., Salpeter, E. E.., \& Stahler, S. W. 1983, ApJ, 271, 632

58. Larson, R. B. 2003, Rep. Prog. Phys., 66, 1651

59. Omukai, K., \& Nishi, R. 1998, ApJ, 508, 141

60. Omukai, K., \& Inutsuka, S. 2002, MNRAS, 332, 59

61. Wolfire, M. G., \& Cassinelli, J. P. 1987, ApJ, 319, 850

62. Perna, R., \& Loeb, A. 1998, ApJ, 501, 467

63. Waxman, E., \& Draine, B. T. 2000, ApJ, 537, 796

64. Fruchter, A., Krolik, J. H., \& Rhoads, J. E. 2001, ApJ, 563, 597

65. Draine, B. T., \& Hao, L. 2002, ApJ, 569, 780

66. MacFadyen, A. I., Woosley, S. E., \& Heger, A. 2001, ApJ, 550, 410 